\title{
Síndrome de rabdomiolisis e hipertermia secundario al consumo de cocaína y/o éxtasis. Comunicación de dos nuevos casos y revisión del problema
}

\author{
E. VALLINA ÁLVAREZ, R. SUÁREZ ARIAS, A. GARCÍA DIEZ, J. M. ARRIBAS \\ CASTRILLO
}

Servicio de Medicina Interna I del Hospital Central de Asturias. Departamento de Medicina Universidad de Oviedo

RHABDOMYOLISIS SYNDROME AND HYPERTHERMIA SECON DARY TO COCAINE AND/OR ECSTASY INTAKE. COMMUNICATION OF TWO NEW CASES AND REVIEW OF THE PROBLEM

\section{RESUMEN}

Se describen dos casos de síndrome de hipertermia y rabdomiolisis en pacientes que habían consumido cocaína y/o éxtasis. Ambos muestran una clínica y unas alteraciones analíticas características de este síndrome. El primer paciente a consecuencia de la toma de cocaína, éxtasis y alcohol sufre un cuadro grave de hipertermia y rabdomiolisis asociado a estupor, confusión, tensión arterial lábil, grave insuficiencia renal, que precisó de hemodiálisis, e intenso daño hepático. El segundo consume éxtasis asociado a alcohol e importante ejercicio físico y sufre de un cuadro similar aunque moderado que revirtió con medidas de reposición hidrolectrolítica. Si bien la incidencia de esta patología es muy limitada en relación al amplísimo consumo de estas drogas, su continuo uso llevará consigo la atención de nuevos pacientes afectos de esta y otras complicaciones agudas. Con este motivo se realiza una revisión y actualización del problema.

PALABRAS CLAVE: Hipertermia. Rabdomiolisis. Cocaina. Éxtasis (metanfetaminas).

\begin{abstract}
We describe two cases of hyperthermia and rhabdomyolysis related to cocaine and/or ectasy consumption. The clinical and laboratory fin dings are characteristic of hyperthermia syndrom in both cases. The first patient consumed cocaine, ecstasy and alcohol and afterwards suffers from hyperthermia and rhabdomyolysis associated with decreased con cious level, labile blood pressure, severe renal insufficiency (needing hemodyalisis) and important liver failure. The second patient consumed ecstasy and alcohol with intense physical activity and shows the same but moderate clinical picture rapidly responding to intrevenous fluid the rapy. The incidence of this drug-induced hyperthermia is low compared to the important use of these illicit drugs. Nevertheless its increasing consumption will lead physicians to attend more patients with this syndrom and other related complications. For this reason we have reviewed and up-dated this problematic subject.
\end{abstract}

KEY WORDS: Hyperthermia. Rhabdomyolysis. Cocaine. Ecstasy (metamphetamines).

Vallina Álvarez E, Suárez Arias R, García Diez A, Arribas Castrillo JM. Síndrome de rabdomiolisis e hipertermia secundario al consumo de cocaína y/o éxtasis. Comunicación de dos nuevos casos y revisión del problema. An Med Interna (Madrid) 2002; 19: 85-88.

\section{INTRODUCCIÓN}

Hasta el año 1960 se conocía como causa fundamental de hipertermia el golpe de calor en sus dos formas, la clásica y la secundaria a ejercicio físico en ambiente de alta temperatura. Está caracterizado clínicamente por un aumento de la temperatura corporal y la asociación de rigidez muscular, rabdomiolisis, insuficiencia renal y otras posibles complicaciones como la coagulación intravascular diseminada, arritmias, asistolia y acidosis metabólica. A partir de la citada fecha, en que se comunica por primera vez un caso de hipertermia maligna (1) causado por anestesia con suxametonio y halotane, se describen nuevos síndromes de hipertermia secundarios al uso de otros medicamentos o drogas. Surgen de este modo, además de la hipertermia maligna, el denominado síndrome neurolép- tico maligno (SNM) debido al manejo de estos medicamentos y la hipertermia secundaria a drogas.

Los estudios en el hombre y en el cerdo han demostrado en la hipertermia maligna un origen genético de tipo autosómico dominante, el valor de las pruebas musculares in vitro como marcadores de susceptibilidad y una alteración bioquímica esencial: el aumento de los iones de calcio en las células musculares. En relación con este hallazgo el tratamiento específico con dantroleno ha logrado modificar satisfactoriamente el pronóstico (2).

Progresivamente, a partir de 1970, se producen sucesivas comunicaciones sobre el SNM secundario al tratamiento psiquiátrico con neurolépticos. Aunque es un síndrome relativamente raro aparece en la literatura con una frecuencia progresiva, incluso en series relativamente amplias. Estudios en

Trabajo aceptado: 27 de marzo de 1998

Correspondencia: J. M. Arribas Castillo. Servicio de Medicina Interna. Hospital Central de Asturias. C/. Celestino Villamil, s/n. 33006 Oviedo 
humanos y experimentales confirman como mecanismo desencadenante la inhibición de los receptores de la dopamina, que sería responsable de alteraciones extrapiramidales (rigidez muscular y rabdomiolisis) y de una disfunción hipotalámica, causa a su vez de la alteración de la capacidad de regulación adecuada de la pérdida de calor (3).

Más recientemente se refieren esporádicamente patologías similares al SNM secundarias a la cocaína. Desde 1985, coincidiendo con la explosión en la utilización de derivados anfetamínicos (éxtasis y otros derivados), aumentan progresivamente los casos motivados por drogas, aunque la incidencia es escasa en relación a su amplísimo consumo.

La presente comunicación tiene por objeto aportar dos nuevos casos de hipertermia, rabdomiolisis, insuficiencia renal, confusión y daño hepático debidos a la toma de cocaína y/o éxtasis y, con este motivo, realizar una actualización del problema.

\section{CASOS APORTADOS}

Caso1. Paciente de 41 años que llega a Urgencias a causa de una intoxicación medicamentosa

Antecedentes personales. No alergias a fármacos. Separado. Fumador de 2 paquetes al día, consumidor ocasional de cocaína, derivados anfetamínicos y cannabis y alcohólico crónico a tratamiento con antabús. No hipertensión arterial, no diabetes ni dislipemias. Ha sido diagnosticado de depresión y sometido a tratamiento con fluoxetina y midazolan.

Enfermedad actual. Después de varios días de consumir cocaína y codeína, ésta a consecuencia de un cuadro catarral, el día anterior, además de alcohol, consumió a lo largo de la noche cocaína, éxtasis y cannabis. En la mañana siguiente fue encontrado "muy dormido", por lo que es trasladado en UVI móvil al hospital.

Exploración física. Temperatura al ingreso $36{ }^{\circ} \mathrm{C}$. TA 105/70. Muy somnoliento, aunque colaborador. Estertores crepitantes pulmonares basales bilaterales. Resto de la exploración normal.

Pruebas complementarias. 18.000 leucocitos (87\% polinucleares, $9,8 \%$ linfocitos), series roja y plaquetaria normales. Coagulación: índice de protrombina 50\%, TTP y fibrinógeno normales. Bioquímica: CPK 3.399 UI, CK-MB 51UI, creatinina 2,7 mg/dl, urea 43 $\mathrm{mg} / \mathrm{dl}$, potasio $5,9 \mathrm{mEq} / \mathrm{L}$, GOT 5.871 UI, GPT 4.621 UI, FA, GGT y bilirrubinemia normales, ácido úrico $12,5 \mathrm{mg} / \mathrm{dl}$. Serologías de hepatitis B y C y de HIV negativas. Gases arteriales normales. LCR normal. ECG: ritmo sinusal a $84 \mathrm{p} / \mathrm{min}$ sin signos de isquemia. Rx tórax al ingreso normal. Análisis de tóxicos en orina positivos para cannabis, opiáceos, cocaína y anfetaminas.

Evolución. Durante los días siguientes tiene una temperatura oscilante entre 38 y $39^{\circ} \mathrm{C}$, estado de confusión y somnolencia, que con posterioridad desaparecieron. La TA se mantuvo en niveles algo bajos (90-100/60-70 $\mathrm{mm} \mathrm{Hg}$ ) hasta el $5^{\circ}$ día, en que hace una crisis hipertensiva, que remitió pronto con tratamiento, La transaminasas mejoraron rápidamente, llegando al séptimo día a valores de 650 la TGO y de 663 la TGP. La CPK ascendió llegando a 7.342 U. Junto a ello desarrolló un cuadro de oligoanuria elevándose las cifras de creatinina hasta valores de $8,9 \mathrm{mg} / \mathrm{dl}$, aumentando los crepitantes bilaterales. En una nueva radiografía de tórax se observan imágenes compatibles con edema pulmonar. Con este motivo se pautaron sesiones de hemodiálisis, con lo que se logró una recuperación funcional renal con diuresis en las ulteriores 24 horas de alrededor de 1.200 cc., aunque persistía alta la creatinina. Al mismo tiempo desaparecieron los estertores, se normalizó la radiografía pulmonar y se asistió a un rápido descenso de los niveles de CPK. En este momento el paciente solicitó el alta voluntaria. No acudió a posteriores revisiones.
Caso 2. Paciente de 31 años que es conducido al Servicio de Urgencias a causa de un cuadro de confusión y agitación.

Antecedentes personales. No alergias medicamentosas conocidas. Traumatismo craneoencefálico hace años. Intervenido de nariz y dedo de mano izquierda tras traumatismos. Consumo esporádico, los fines de semana, de speed (éxtasis) y alcohol. Enfermedad actual. Acude a urgencias porque después de jugar un partido de rugby sufre un cuadro de confusión, agitación, agresividad, desorientación y vómitos. Se le administra diazepán i.m.y se traslada al hospital donde ingresa consciente, orientado y colaborador, sin recordar nada de lo acontecido. No parece haber recibido ningún traumatismo ni pérdida de conciencia. Los acompañantes refieren que ha dormido poco los días previos y la noche anterior al partido había consumido alcohol y speed.

Exploración física. Consciente, orientado, colaborador. Temperatura $39^{\circ} \mathrm{C}$. Lenguaje normal en contenido y fluencia. Exploración neurológica normal. No signos meníngeos. Orofaringe ligeramente enrojecida. Auscultación cardiaca normal, a 80 latidos/minuto y soplo sistólico II/VI panfocal. Tensión arterial 120/80 mm de Hg. Exploraciónes pulmonar, abdominal y de pulsos periféricos normales.

Estudios complementarios. Leucocitos 32.500 (75\% polinucleares y $3 \%$ cayados), $\mathrm{Hb} 17,1 \mathrm{~g} / \mathrm{dl}$, volúmenes corpusculares normales, plaquetas 325.000. Coagulación normal. Urea 73,8, creatininemia 2,5, CPK 1.529 (fracción MB normal), Brb total 2,13, Brb D 0,6, TGO 200, TGP 271, LDH 616, aldolasa 17,9, glucosa 112 mg/dl. Resto de parámetros analíticos incluyendo FA, GGT, colinesterasa, amilasa, perfíl lipídico, iones y proteinanograma normales. Serologías negativas para CMV, brucela, fiebre Q, virus de la hepatitis B y C y VIH. IgG positivas para EBV y Mycoplasma. Orina con sedimento 48-50 hematíes, 2-4 leucocitos y escasa flora bacteriana. Gasometría arterial basal normal. LCR normal. ECG: ritmo sinusal a 110/minuto. TAC craneal: pequeña imagen hipodensa frontal derecha en relación con contusión a dicho nivel sin signos de hemorragia (antecedente de traumatismo años antes). Ecografía abdominal normal. Hemocultivos y urinocultivos negativos.

Evolución. Se instauró sueroterapia. Recuperó en unos días los valores de la función renal. Descendieron los valores de transaminasas y persistieron elevados los de CPK, aunque con tendencia al descenso. Se dio de alta al cabo de cinco días del ingreso.

\section{DISCUSIÓN}

El primer paciente, tras la toma días antes de cocaína y codeina, consumió la noche anterior a su ingreso cantidades importantes de alcohol, cocaína, éxtasis y cannabis. Como consecuencia de ello sufrió un cuadro clínico caracterizado por sueño profundo seguido de confusión y somnolencia, hipertermia, rabdomiolisis, insuficiencia renal, leucocitosis e intenso daño hepático. Presentó los primeros días una hipotensión moderada y desarrolló al $5^{\circ}$ día una crisis hipertensiva. Todas estas manifestaciones son comunes en el cuadro de hipertermia del síndrome neuroléptico maligno, cumpliendo los criterios establecidos para su diagnóstico $(4,5)$ excepto el antecedente de la toma de neurolépticos, la ausencia de rigidez muscular (dato mayor de los criterios) y los signos de daño hepático (no citados en ellos). El cuadro clínico del segundo paciente también incluyó la hipertermia, el estado de confusión y agitación, rabdomiolisis, leucocitosis, una moderada insuficiencia renal, pronto reversible, y datos analíticos de daño hepático. Tampoco mostró rigidez muscular. En él son hechos a tener en cuenta la ingesta previa de alcohol y éxtasis, la práctica de un ejercicio físico intenso y, consecuentemente, la sudoración con pérdida de agua y electrolitos. 
En relación a la cocaína se ha observado un mayor número de muertes y accidentes de tráfico por sobredosis en periodos de alta temperatura, superior a $30^{\circ}$, así como de los cuadros de rabdomiolisis e hipertermia (6). En la descripción de la literatura los pacientes afectados de rabdomiolisis (7) asociada a otras complicaciones de las referidas han recibido la droga por cualquiera de las vías de administración oral, nasal, intravenosa o en forma de "Crack" sin clara relación con la forma de administración y la dosis. Como ejemplo, en la serie publicada por Daras y cols, (8). de 14 pacientes, 7 utilizaron "Crack", 2 la vía venosa y 3 la vía nasal. En relación con la clínica y el pronóstico, en todos existía rabdomiolisis, en 13 hipertermia, en 11 alteración del estado mental, en 11 elevación importante de las transaminasas, en 9 fallo renal, en 8 taquicardia y TA lábil, en 6 coagulación intravascular diseminada y solo en 4 rigidez muscular. Fallecieron 5 enfermos, 3 con insuficiencia renal y 2 por parada cardiorespiratoria. Como ocurrió en nuestro primer paciente, todos ofrecían niveles elevados de ácido úrico.

Por otra parte se han descrito esporádicamente tras la utilización de cocaina otras complicaciones de gravedad, no asociadas necesariamente a rabdomiolisis e hipertermia, como cuadros agudos coronarios, infartos de miocardio sin obstrucción ni trombosis (debidos fundamentalmente a un mecanismo vasoconstrictor) $(9,10)$, arritmias, hemorragias cerebrales y convulsiones (11). Recientemente se ha demostrado la facilitación de abortos en pacientes consumidoras de cocaina y alcohol (12).

Síndromes similares a los descritos en nuestros pacientes son continuamente referidos en la literatura de estos años a consecuencia del consumo de éxtasis y otras metanfetaminas (13), aunque las series son pequeñas y la mayoría comunicaciones de casos únicos. Como ocurrió con nuestro segundo paciente son factores facilitadores comunes el alcohol, el calor ambiental y un importante ejercicio físico, bien deportivo o en forma de baile intenso (14). Con frecuencia causan, aunque pasajeramente, alucinaciones y trastornos en la percepción.

Mención especial merece la consideración de la afectación hepática. La cocaína es causa de importante daño hepático, a menudo grave, asociado a rabdomiolisis. Los pacientes que cursan con el síndrome rabdomiolisis, hipertermia y daño hepático grave ofrecen un peor pronóstico. En la serie publicada por Silva y cols, (15) de 39 pacientes afectados de rabdomiolisis por consumo de cocaína mostraron datos bioquímicos de disfunción hepática 23(59\%). De ellos, 16 tenían un nivel de ALT superior a $400 \mathrm{U} / \mathrm{L}$ y fallecieron 7 (44\%). Los estudios histopatológicos postmorten evidenciaron la existencia de una necrosis extensa centrolobulillar y zonal media. En dos de ellos la necrosis era panlobular. La lesión hepática como hecho aislado, no asociado a rabdomiolisis, es excepcional en el hombre $(16,17)$ (aunque habitual en la experimentación animal en ratas) $(18,19)$.

El daño hepático sin rabdomiolisis es más frecuente a consecuencia del consumo de metanfetaminas. Junto a comunicaciones de casos individualizados se han publicado en esta década algunas series, aunque con un número de pacientes corto. El daño hepático puede surgir como consecuencia del consumo de éxtasis o de productos de acción similar en tres formas de presentación:

1. Asociado a rabdomiolisis y manifestaciones acompañantes (20), como en nuestros pacientes.
2. Sin rabdomiolisis, de aparición inmediata o horas después del consumo.

3. Sin rabdomiolisis y de aparición más tardía con un intervalo de varios días o semanas (21-23).

En las dos primeras formas de expresión los estudios histopatológicos muestran datos de necrosis como los referidos a propósito de la cocaína. En la forma más tardía ofrece un patrón clínico y anatomopatológico similar al de la hepatitis aguda.

Resulta concluyente que, ante todo cuadro de fallo hepático agudo, descartadas la hepatitis víricas, el consumo de metanfetaminas ocupa un lugar muy importante como factor etiológico en la población joven. En la serie publicada por Andreu y cols. (22), constituía el $20 \%$ de los pacientes ingresados en la Unidad de Cuidados Intensivos de Hepatología del Hospital Clínico de Barcelona por fallo hepático agudo en edades inferiores a 25 años. En la serie de Ellis y cols, (21), de ocho enfermos que ingresaron por su consumo en la Unidad de Hepatología del Instituto de Estudios del Hígado de Londres dos padecieron de daño hepático agudo asociado a rabdomiolisis e hipertermia, cuatro presentaron un fallo agudo sin hipertermia (cumplían criterios de transplante y tres de ellos fallecieron) y otros dos sufrieron de hepatitis aguda con recuperación en unas semanas.

Sin bien de lo expuesto puede derivarse la escasa frecuencia de las referidas complicaciones agudas a consecuencia del consumo de cocaína y/o anfetaminas, su dilatado consumo actual lleva a la deducción de que seguirán surgiendo en los años venideros. Baste citar algunos datos al respecto. Treinta millones de americanos han experimentado con cocaína y/o anfetaminas y más 7,5 millones son consumidores habituales (24). En nuestro país en el año 1987 se decomisaron 187 pastillas de MDMA (éxtasis) y en 1994 306.501, siendo las regiones de mayor consumo las del litoral mediterráneo, Baleares y Navarra (25). Por otra parte, encuestas realizadas a varios reemplazos del servicio militar en los años 1994 a 1997 demuestran que las habían probado alguna vez entre el 8,6 y el 13\%. La edad media de iniciación era de 17 años (26).

La cocaína se consume en dos formas distintas: a) el hidroxicloruro, soluble en agua, que se puede utilizar como spray nasal, por vía oral o intravenosa; y b) el alcaloide, insoluble en agua y resistente al calor, conocido por los términos de Crack o Rock, como tabaco.

El efecto de estos preparados dura aproximadamente 90 minutos, independientemente de la vía utilizada.

Las metanfetaminas, conocidas con el término de "cristal", se utilizan habitualmente en forma de comprimidos por vía oral. Si bien la más habitual es la 3,4, metilendioximetanfetamina, MDMA, conocida como éxtasis, se han ido incorporando derivados de acción similar como el MDA (píldora del amor), el MDEA (Eva) y más recientemente otros como el DOB y MBDB. Las metanfetaminas tienen un tiempo de acción más largo, son más fáciles de producir y resultan más baratas, por lo que su consumo, fundamentalmente como droga de las fiestas de fin de semana, llega a superar al de la cocaina. Por ello la mayoría de sus consumidores lo son de tipo social con utilización discontinua. Las píldoras suelen estar adulteradas y contener, además de la metanfetamina, otros componentes añadidos como cafeina (prácticamente siempre), testosterona, estimulantes (como pseudoepinefrinas o drogas psicoactivas), opiáceos y cocaína, por lo que plantean la duda de hasta qué punto la acción tóxica debe imputarse exclusivamente a la metanfetamina (27). 
La cocaina y las metanfetaminas producen sus efectos determinantes del síndrome de rabdomiolisis e hipertermia por elevar las concentraciones sinápticas de norepinefrina, dopamina y serotonina. Las metanfetaminas causan un aumento de su liberación presináptica e inhiben su recaptación. También impiden encimáticamente la síntesis de 5-HT (28). Debido a la depleción de serotonina, los consumidores crónicos padecen con gran frecuencia una amplia variedad de síndromes psiquiátricos (depresión, cuadros psicóticos, alteraciones del conocimiento, episodios de bulimia, de pánico o fobias), como se comprueba en pacientes sometidos a tratamientos de deshabituación $(29,30)$. La cocaina da lugar a efectos parecidos al inhibir la recaptación postsináptica de los tres neurotransmisores.
Las consecuencias son una disfunción central secundaria a la activación de los correspondientes receptores, que afecta al hipotálamo, area límbica y cortex y cambios periféricos. En la periferia dos mecanismos son causa a la vez de la hipertermia: por un lado el aumento en la génesis de calor y por otro la pérdida de su disipación, debido a la vasoconstricción. A nivel central dan lugar a cambios funcionales que repercuten en las esferas psíquica y cardiocirculatoria y en la regulación térmica hipotalámica. Diferentes investigaciones en experimentación animal, mediante el estudio del efecto de agonistas y antagosnistas de los receptores de dopamina y 5-HT, demuestran su participación directa así como una coordinación entre la acción de los receptores de dopamina y de 5-HT.

\section{Bibliografía}

1. Denborough MA, Lovell RRH. Anaesthetic deaths in family. Lancet, 1960; 2: 45.

2. Denborough MA. Hipertermia maligna. Lancet (Ed. Esp). The Lancet, 1998; 352: 1131-1136

3. Ronald J, Gurrera, Sydney S. Chang. Thermoregulatory Dysfuntion in Neurologic Malignant Syndrome. Biol Phych 1996; 39: 207-212.

4. Varadaraj R. Velamor. Neuroleptic Malignant Syndrome. Recognition, Prevention and Management. Drg Safety, 1998; 19(1): 73-82.

5. Ronald J, Gurrera MD, Sidney S, Cahng MD, Jorge A, Romero MD. A Comparation of Diagnostic Criteria for Neuroleptic Malignant Syndrome. J Clin Psychiatry 1992; 53(2): 56-61.

6. Marzuk PM, Tardiff K, Leon AC, Hirsch CS, Portera L, Iqbal MI, et al. Ambiente temperature from unintentional cocaine overdose. JAMA, 1998; 279(22): 1795-1800.

7. Sánchez Navarro MR, Wangensteen-Fuentes R, Oliver Almendros C. Isoformas de creatin quinasa-MM e isoenzimas de lásticodeshidrogenasa en el suero de pacientes con rabdomiolisis aguda no traumática. An Med Interna (Madrid) 1998; Vol 15, No 1: 13-17.

8. Daras M, Kakkouras L, Tuchman AJ, Koppel BS. Rhabdomyolysis and Hyperthermia After Cocaine Abuse: a variant of the neuroleotic malignant syndrome? Acta Neurol Scand 1995; 92(2): 161-165

9. Batalla Celorio A, Iglesias Cubero G, Rodriguez Reguero JJ,et al. Infarto de miocardio con consumo de cocaina. An Med Int 1999; 16 : $189-190$.

10. Ayala I, Altieri PI. Cocaina induced myocardial ischemia. PR Health Sci J 1993; 12(1): 73-75.

11. Myers JA, Earnest MP. Generalized seizures and cocaine abuse. Neurology 1984; 34(5): 675-676.

12. Roberta B. Ness MD, Jeane Ann Grisso MD, Nancy Hirschinger, MD and cols. Cocaine and tobacco use and the risk of spontaneous abortion. N Engl J Med 1999; 340: 333-339.

13. Theodore C, Chan MD, Shawun D, Evans MD, Richard F, et al. Droginduced hypertermia. Medical toxicology 1997; 23(4): 785-808.

14. Ramcharan S, Meenhorst PL, Otten JM, et al. Survival after massive extasis overdose. J Toxicol Clin Toxicol 1998; 36(7): 727-731.

15. Silva MO, Roth KR, Fernandez JA, et al. Hepatic dysfuntion accompanying acute cocaine intoxication. J Hepatol 1991; 12(3): 312-315.

16. Gubbins CP, Schiffman RM, Alapati RS, Batra SK. Cocaine-induced hepatotoxicidad: a case report. Henry Ford Hosp Me J 1990; 38(1): 55-56.

17. Perino LE, Warren GH, Levine JS. Cocaina induced hepatoxicity in humans. P R Health Sci J 1993; 12(1): 73-75.

18. Kanel GC, Cassidy W, Shuster L, Reynolds TB. Cocaine-induced liver cell injury: Comparison of morphological features in man and in experimental models. Hepatology 1990; 11(4): 646-651.

19. Salminen WF Jr, Robers SM, Fenna M, Voellmy R. Heat shock protein induction in murine liver after acute treatment with cocaine. Hepatology 1997; 25(5): 1147-1153.

20. Rabdomiolisis masiva tras sobredosis de drogas intravenosas complicada con fracaso renal agudo y síndrome compartimental generalizado. An Med Interna (Madrid) 2000; Vol 17, Nº 8: 449-450.

21. Ellis AJ, Wendon JA, Portmann B, Williams R. Acute liver damage and ecstasy ingestion. Gut 1996; 38(3): 454-458.

22. Andreu V, Bas A, Bruguera M, et al. Ecstasy: a common cause of severe acute hepatotoxicity. J Hepatol 1998; 29(3): 394-397.

23. Fidler H, Dhillon A, Gertner D, Burroughs A. Chronic ecstasy (3,4-met hilenedioxymetamphetamine) abuse: a recurrent and unpredictable cause of severe acute hepatitis. J Hepatol 1996; 25(4): 563-566

24. Theodore C, Chan MD, Shawn D, Evans MD, Richard F, Clark MD. Drug-induced hyperthermia. Critical Care Clinics.1997; 13(4): 785-808.

25. de la Fuente de Hoz L, Rodríguez Arenas $\mathbf{M}^{\mathrm{a}} \mathrm{A}$, Vicente Orta J, Sánchez Payá J, Barrio Anta G. Epidemiología del consumo de drogas de diseño en España. Med Clin (Barc) 1997; 108: 54-61.

26. Bobes García J, Saiz Mmartínez PA, Bascarán Fernández MT, Fernández Miranda JJ, López Rodríguez JL. Aproximación epidemiológica y tendencias del consumo de MDMA. Extasis (MDMA): Un abordaje comprehensivo. Editorial Masson.1998.

27. Giroud C, Augsburger M, Sadeghipour F, Varesio E, Veuthey J1, Rivier L. Ecstasy-the status in Frech-speaking Swizerland. Composition of seized grugs, analysis of biological specimens and short review of its pharmacological action and toxicity. Schweiz Rundsch Med Prax 1997; 86(13): 510-523

28. Penning EJ, Konijn KZ, de Wolff FA. Clinical y toxicologic aspects of the use of Ecstasy. Ned Tijdschr Geneeskd, 1998; 142(35): 1942-1946.

29. Schifano F, Di Furia L, Forza G, Minicuci N, Bricolo R. MDMA (ecstasy) consumption in the context of polydrug abuse: a report on 150 patients. Drug Alcohol Depend 1998; 52(1): 85-90.

30. Nielsen JC, Nicholson K, Pitzner-Jorgensen BL, Unden M. Abuse of Ecstasy. Pharmacological, neuropsychiatric and behavioral aspects. Ugeskr Laeger 1995; 157(6): 724-727.

31. Rockhold RW, Carver ES, Ishizuca Y, Hoskins B. Dopamine receptor mediate cacaine-induced temperature responses in spontaneusly hypertensive and wistar-kyoto rats. Pharmacol Biochem Benay 1991; 40:157.

32. Salmi P, Anhelius S. Evidence for functional Interactions between 5HT1 and 5-HT2A receptors in rat thermoregulatory mechanisms. Pharmacol Toxicol 1998; 82(3): 122-127.

33. Yamawaki S, Lai H, Horita A. Dopaminergic and serotonergic machanissms of thermoregulation: Mediation of thermal effects of aphomorphine and dopamine. J Pharmacol Exp Ther 1983; 227-383. 